\title{
Element Ratio Maps: A More Informative Use Of Digital Elemental X-ray Maps Than Single Element Maps
}

\author{
C.S. Schwandt and G.A. McKay \\ Astromaterials Research Office, Johnson Space Center, Code KR, Houston, TX 77058 USA
}

Over the past few years the acquisition of analytical data has become an entirely digital process. Several advantages result from this improvement, primarily the ability to collect copious amounts of high quality information. Coupled with advances in instrumentation such as improved specimen stage control and positioning and improved control of electron beam diameter, coherence, and intensity, rapid data collection facilitates wonderful new research discoveries. However, these advances are often not fully utilized when data examined with conventional historical methods because the data sets are extremely large and can be difficult to work with using typical personal computer (PC) hardware and software. As a result of both the large size of the data sets and conventional thinking, some scientists end up essentially throwing away portions of the valuable information they collected by converting their data into simpler forms, such as image file formats $[1,2]$. Other scientists are developing innovative methods based on geostatistics which allow for the rapid inspection and analysis of the specimen using the entire digital data set. This approach allows for discovery of unsuspected constituents within the specimen whether the investigator knows anything about the specimen before analysis or not $[2,3,4]$. Although this later approach is extremely powerful, many investigators do know something about their specimen prior to analysis. Therefore the investigator can select the appropriate components ahead of time, effectively conducting a principal component analysis without actually churning through the calculations. This pre-selection of components can drastically reduce the size of the data cube ( $\mathrm{x}$ and $\mathrm{y}$ spatial coordinates by $\mathrm{n}$ number of components or channels), thus drastically reducing the computational overhead and, more importantly, allowing the computational savings to be applied to the spatial resolution and size parameters of the data cube. Two key facilitating factors which make the approach described here powerful are using the native X-ray intensity data in its raw form and manipulating it mathematically with a crystal chemical concept using a computer programming language optimized for handling large data cubes.

The crystal structure of minerals places constraints on the relative number and locations of component elements within a unit cell (the smallest structure that uniquely identifies the mineral or phase). Therefore one can utilize the ratio of cation elements occupying crystal structure positions coordinated by six oxygen atoms (octahedral coordination) to the cation elements occupying crystal structure positions coordinated by four oxygen atoms (tetrahedral coordination), as well as elements unique to specific phases, to sort out and categorize the unique constituents of the specimen. These unique phases can then be easily visualized with synthesized color images. The ratios can be mathematically tuned to provide unique distinctions between the specimen constituents. These mathematical visualizations can provide petrologic or formational insights that might otherwise be lost by examination of individual component (i.e., element) maps. Importantly, many specimen constituents do not consist simply of end-member compositions but are solid-solution constituents (a mixture of cation elements occupying the same general crystal structure position). Using this octahedral to tetrahedral element ratio 
concept thus allows for the unique separation of constituents where otherwise examination of single component visualizations would be non-unique because multiple constituents may have similar ranges of element or component concentrations (i.e. X-ray intensities).

One perceived disadvantage of this approach is that using stage mapping (rastering the specimen stage beneath a fixed electron beam) causes significantly more electron beam damage than a scanning acquisition with the spectral imaging approach. However, ample results are achieved using an accelerating voltage of $15 \mathrm{kV}$ and a probe current of $40 \mathrm{nA}$ with a pixel dwell time of at least $30 \mathrm{~ms}$. These condition typically yield peak to background X-ray intensity ratios of at least ten to one for major constituent elements. Collection of ten elements provides the most latitude with the stoichiometric mathematic combinations. For a 5-spectrometer instrument, this leads to a combined beam exposure time per pixel of only $60 \mathrm{~ms}$. (When trace elements are important, obviously longer dwell times are required to achieve adequate X-ray intensities.) Therefore, stage mapping need not be more damaging to specimens than the spectral imaging approach.

Our research group utilizes the approach described here to determine percentages of the different mineral phases in meteorite thin sections (Fig. 1). The percentages are used with quantitative analyses of the phases to calculate an overall bulk chemical composition of the meteorite. Additional petrologic aspects are inspected with the octahedral cation over tetrahedral cation maps. Removing the confusion can occur with single element maps due to concentration overlaps for solid solutions with back-scattered electron images due to low contrast difference between phases provides insights that are not otherwise obvious.

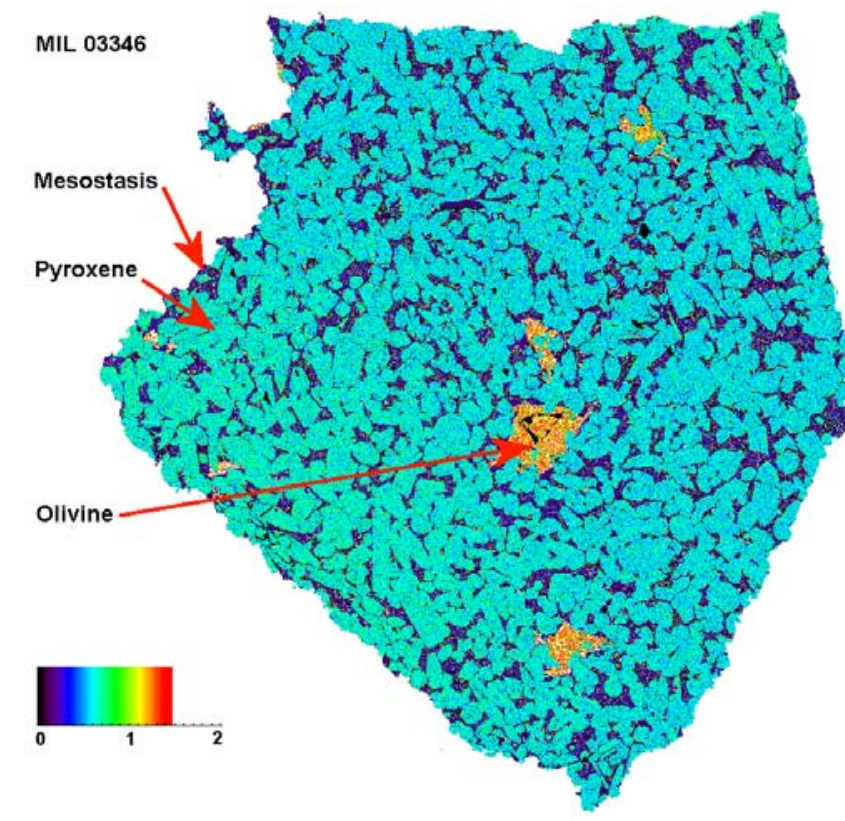

Figure $1(\mathrm{Mg}+\mathrm{Fe}+\mathrm{Ca}) / \mathrm{Si}$ map. Thin section is $1.6 \mathrm{~cm}$ wide, represented by $5,822,756$ pixels.

\section{References}

[1] T.L. Hicks et al., Lunar and Planetary Science XXXI (CD-ROM), (2000) \#1491. [2] T.L. Hicks et al., Lunar and Planetary Science XXXIII (CD-ROM), (2002) \#1055. [3] P.G. Kotula el al., Microsc. Microanal. 10(Suppl 2) (2004) 118.

[4] M.R. Keenan and P.G. Kotula., Microsc. Microanal. 10(Suppl 2) (2004) 874.

[5] P.G. Kotula el al., Microsc. Microanal. 10(Suppl 2) (2004) 892.

[6] This work was supported by NASA RTOP 344-31-20-23 to Gordon McKay. 